\title{
Flexible Memory Processing by Rats: Use of Prospective and Retrospective Information in the Radial Maze
}

\author{
Robert G. Cook, Michael F. Brown, and Donald A. Riley \\ University of California, Berkeley
}

\begin{abstract}
Four experiments investigated the content of the memory used by rats in mediating retention intervals interpolated during performance in a 12-arm radial maze. The delay occurred following either the 2 nd, 4 th, 6 th, 8 th, or 10 th choice. A 15-min delay had the greatest disruptive effect when interpolated in the middle of the choice sequence and less of an effect when it occurred either earlier or later. This pattern of results was obtained when either a free- or forced-choice procedure was used prior to the delay and regardless of whether postdelay testing consisted of completion of the maze or two-alternative forced-choice tests. Assuming that the disruptive effect of a delay is a function of memory load, this implies that the rats used information about previously visited arms (retrospective memory) following an earlier interpolated delay but information about anticipated choices (prospective memory) following a delay interpolated late in the choice sequence. There appeared to be a recency effect only in the early and middle delay conditions. This provides converging evidence for the dual-code hypothesis. No evidence for prospective memory was obtained following a 60 -min delay.
\end{abstract}

Many conceptions of animal short-term or working memory assume that previously experienced events are represented in memory

The contributions of the first two authors to this project were equal, and their order of authorship was determined by lot.

Portions of these data were presented at the April, 1982, meeting of the Western Psychological Association and the November, 1984, meeting of the Psychonomics Society.

Support during the course of the experiments was provided by Biomedical Research Support Program Grant 2-S07-RR07006 from the Division of Research Resources, National Institutes of Health, and by National Science Foundation (NSF) Grant BNS-7908839 to D. A. Riley.

Additional support during preparation of the manuscript was provided by NSF Grant BNS-8317577 to D. A. Riley and National Research Service Award Postdoctoral grants from the National Eye Institute, Grants 5 T32 EY-07024 and I FY EY-05653-01A1 to R. G. Cook.

Many people provided valuable advice and discussions during the course of this work and the preparation of this manuscript; we would like to give particular thanks to Alan Bond, Ervin Hafter, Barbara Mellers, Seth Roberts, John Watson, Tom Wickens, Sonja Yoerg, and several participants in the annual Berkeley-Stanford Cognitive psychology meetings.

We also thank Steve Heinrichs and George Kim for their help in running Experiments 3 and 4.

Requests for reprints should be sent to Robert $G$. Cook, University of Texas Health Science Center, 6420 Lamar-Fleming Boulevard, Texas Medical Center, Houston, Texas 77030 . (e.g., Roberts \& Grant, 1976). Several investigators have recently proposed, however, that anticipated future events may also be retained in working memory (e.g., Grant, 1981; Honig, 1978, 1981; Honig \& Thompson, 1982; Honig \& Wasserman, 1981; Riley, Cook, \& Lamb, 1981; Roitblat, 1980, 1982; Weisman \& DiFranco, 1981). This idea has emerged from work directed at the processes that allow animals to mediate the delay between a conditional cue and a response in paradigms such as matching-to-sample. The proposition has been that during or shortly after presentation of the conditional cue, information is encoded in a form that is related to the upcoming test stimulus or the response associated with it, rather than to the preceding conditional cue. It has been argued, for example, that in the delayed matching-to-sample paradigm, pigeons remember the nature of the correct test stimulus during the delay rather than the previously experienced sample stimulus (Roitblat, 1980). Honig (1978) suggested that the animal remembers prospective instructions (what to do) rather than retrospective information (what it saw). Riley, Cook, and Lamb (1981) argued that evidence for three different types of memory coding can be found: prospective coding of anticipated stimuli, retrospective coding of previ- 
ously experienced stimuli, and response mediation in which neither type of information need be represented in memory.

The present experiments were designed to investigate memory coding in the radial arm maze. In the radial maze, rats are placed on a central elevated platform from which a number of arms radiate. Each of the arms is baited with a small amount of food, and the task is to deplete the food from the arms. Revisits to arms that have been visited previously in the trial are considered errors because food is no longer available. Previous work has shown that rats make very few errors in an eight-arm maze (e.g., Olton \& Samuelson, 1976) and perform above chance in a 17-arm maze (Olton, Collison, \& Werz, 1977). The cues necessary for accurate performance are the extramaze cues associated with the arms rather than odors or response algorithms; that fact makes it clear that performance in this task depends on some form of short-term or working memory system (cf. Olton \& Collison, 1979; Olton \& Samuelson, 1976; Suzuki, Augerinos, \& Black, 1980).

In the present experiments, three hypotheses about the nature of the memory representations used in the radial maze were contrasted. Rats could avoid previously chosen arms and/or find novel arms by (a) maintaining representations of previously visited arms, (b) maintaining representations of anticipated, to-be-visited arms, or (c) both. For example, rats could discriminate previously visited arms from correct ones by maintaining representations of previously visited arms and visiting only arms that are not represented in memory. Alternatively, highly trained subjects, with long-term knowledge or reference memory about the nature of the arms in the maze, could maintain representations of anticipated, to-be-visited arms and delete representations of arms as they visit them, visiting only those arms that remain in memory. The first hypothesis corresponds to retrospective coding, whereas the second corresponds to prospective coding.

In this task, a number of items (arms) must be remembered rather than a single stimulus. This property of the task presents the animal with a situation in which the relative merit of retrospective coding and prospective coding changes over the course of each trial. Early in the trial, there are few visited arms but many unvisited arms. Thus, a retrospective code would contain less information and may be more efficient. As arms are visited, however, prospective memory might become more efficient.

Honig and Thompson (1982) suggested that rats use retrospective coding in the radial maze. The strongest support for their conclusion came from the serial position curves of errors. Several experiments have shown that revisits to choices made early in the choice sequence occur more often than do revisits to choices made late in the choice sequence (e.g., Kessner \& Novak, 1982; Olton \& Samuelson, 1976; Roberts \& Smythe, 1979). Thus, rats seem more likely to avoid revisiting more recent choices than choices made earlier. This recency effect implies that retrospective coding is being used (but see Olton et al., 1977 for an alternative explanation of the recency effects found by Olton and Samuelson, 1976). If prospective memory were being used, then there should be no relation between the order in which arms are visited and the probability of revisiting them, because memory would not contain representations of the arms already visited, but instead would contain representations of anticipated future choices.

The type of memory code used in the maze was examined in the present experiments by varying the location of a delay in the choice sequence. The basic design involved placing rats in a 12-arm maze and allowing them to make $2,4,6,8$, or 10 choices. They were then taken out of the maze for a retention interval before being returned to finish the maze.

Two assumptions allowed behavioral predictions corresponding to the aforementioned coding hypotheses to be made. First, it was assumed that there is a correspondence between the number of arms visited (or remaining) and the amount of information stored in memory. Second, it was assumed that the disruptive effect of a retention interval is greater when memory contains more information than when it contains less information.

Given these assumptions, the three coding hypotheses make unique predictions about how delays should affect accuracy. First, if the rats remember retrospectively, then a 
delay interpolated between choices near the end of a trial (e.g., after Choice 10) should disrupt performance to a greater extent than a delay interpolated earlier (e.g., after Choice 2 ), because the amount of information to remember is greater. If the rats retain prospective information about where to go or which arms still have food, however, then increasingly later interpolation of the delay should result in less disruption because memory load would be less. Third, rats could use both codes and keep memory load at a minimum in all conditions by using whichever code reduces the amount of information to remember during the delay. If the delay occurs early, they could remember the few arms visited rather than the many still remaining. If the delay occurs late, they could remember the few arms that remain. Such a combination would result in less disruption of performance following either an early or late delay than when the delay occurred in the middle of the choice sequence. During a delay in the middle of the choice sequence, neither a short retrospective "list" nor a short prospective list could be used.

The serial position curves of errors, reflecting the relation between the order in which arms are visited prior to the delay and the tendency of rats to revisit them, were also of interest because of their implications for the form in which information is represented in memory. As is well known, humans are able to recall items better if they appear close to the end of a presented list than if they appear in the middle of the list (Murdock, 1962). Such recency effects have also been reported in studies of animal memory (e.g., Sands \& Wright, 1980; Wagner \& Pfautz, 1978), including the radial maze (e.g., Kesner \& Novack, 1982; Olton \& Samuelson, 1976; Roberts \& Smythe, 1979). The major interpretation of the recency effect has been that it reflects the existence of the later items in a limited-capacity short-term storage system (e.g., Atkinson \& Shiffrin, 1968). A more recent interpretation attributes the recency effect to contextually guided retrieval (Glenberg, Bradley, Kraus, \& Renzaglia, 1983). This theory states that the encoding context serves as a retrieval cue and that the greater similarity between the context in which the last few items were encoded and the context at the time of recall results in better recall of the last few items. Both of these theories require retrospective memory as a prerequisite for obtaining serial position effects. Unless the previously presented items are retained in memory, they cannot be avoided differentially due to their presence in a short-term storage system or to the greater similarity of their contextual cues to the context at the time of the test. Thus, if rats are using prospective memory, then no serial position effects are expected, because the information in memory is not in the form of previous choices and, hence, cannot be avoided differentially.

\section{Experiment 1: Variation of the Point-of- Delay Interpolation}

The first experiment used the general strategy outlined above. Rats were allowed to choose from among the 12 arms of the maze until they made either $2,4,6,8$, or 10 choices. They were then removed from the maze and placed in a holding cage for a 15min delay interval, after which they were returned to the maze and allowed to complete the trial.

The subjects' ability to avoid the arms chosen prior to the delay relative to their ability to do so during trials in which no delay occurred was of primary interest. This comparison was the measure of the effect of the delay on the information contained in memory. As was argued above, the shape of the function relating point-of-delay interpolation and the effect of the delay on choice accuracy reveals the nature of the coding process used.

The use of prospective memory would require long-term or reference memory information about the maze, because information about arms that have not yet been chosen is retained during the delay. Therefore, subjects were given extensive experience in the maze prior to being tested.

\section{Method}

Subjects. The subjects were 10 Sprague-Dawley albino rats ( 6 males, 4 females). They were maintained at $80 \%-$ $85 \%$ of their free-feeding weights in a room with a $12: 12$ light-dark cycle and free access to water. Trials occurred during the first $4 \mathrm{hr}$ of their dark phase. Supplemental 
feedings were given immediately following experimental sessions. One rat became ill during the course of the experiment. Its data are not included.

Apparatus. The apparatus was a 12-arm radial maze. It consisted of arms radiating out of a center platform (41 $\mathrm{cm}$ in diameter) at equal angles. The maze was elevated $61 \mathrm{~cm}$ above the floor. Each arm was $80 \mathrm{~cm}$ long and $10 \mathrm{~cm}$ wide. The entrance to each arm was through a $7.5-\mathrm{cm}$ hole in a metal barrier that surrounded the center platform. Entrance to the arms was controlled by a single cylindrical guillotine door. $A$ barrier $(12.5 \mathrm{~cm}$ in height $\times 26.0 \mathrm{~cm}$ in length) was located on the right side of each arm to prevent the animals from visiting the next arm without returning to the center platform. Stainless steel food cups $(10.0 \mathrm{~cm}$ in diameter and 1.5 $\mathrm{cm}$ deep) were located at the end of each arm. The maze was located in a well-illuminated room with a variety of extramaze cues, including a large furnace, a rack of spare cages, a large white screen, a sink, the subjects' home cages, the experimenter, and a large black plastic tarp that divided two sides of the experimental area from the rest of a large room.

During delays, subjects were placed in small, opaque, plastic cages with free access to water. These cages were located approximately $2 \mathrm{~m}$ from the maze. They were distinct from the larger wire-mesh home cages.

Procedure. Subjects were first shaped to enter the arms and eat from the food cups by placing food pellets (Noyes $45 \mathrm{mg}$ ) along each arm and in the cups. Following several sessions of this initial training, each arm was baited with two pellets, and subjects were placed in the maze until all 12 arms had been visited, 20 choices had been made, or $20 \mathrm{~min}$ had elapsed. Training continued for 60 sessions, during which time a number of behavioral measurements were taken (Brown \& Cook, 1982).

Following training, two blocks of 12 trials were conducted. Each block contained six different conditions, run twice per block. In five of these conditions, a 15 min delay was interpolated during the trial. It occurred following either the 2 nd, 4th, 6th, 8 th, or 10th choice of the trial. This factor will subsequently be referred to as point of interpolation (POI). Each block also contained two control trials during which no delay occurred and the subject was allowed to make choices until it had completed the maze or made 20 choices. The order in which conditions occurred was random within each block. Rats were given one trial per day, 6 days a week.

At the beginning of each trial the rat was placed in the center platform in a random orientation, and the guillotine door was raised approximately 10-15 s later. It was allowed to choose freely from among the arms. A choice was defined as moving past the end of the barrier located on each arm. In delay conditions, the guillotine door was closed when the subject reentered the center platform following its last predelay choice. The animal was then placed in its individual holding cage. Cages that were distinct from the home cages were used to differentiate delay periods from the end of a trial (signaled by return to the home cage). After $15 \mathrm{~min}$, the rat was returned to the maze and allowed to finish the trial or allowed to continue until 20 total choices had been made (inclusive of choices made prior to the delay). Only the arms not previously visited were baited at this time. During a subject's delay other rats (1-3) were run in the maze. In the control condition, no delay occurred. The subject was allowed to run in the maze until 12 correct choices or 20 total choices had been made.

Data analysis. A common measure of performance in the radial maze is the number of choices required to finish the maze. Such measures of overall performance are not adequate for comparisons among the various conditions used in the present experiment, however. Whatever effect the delay has, it is restricted to those choices that are made following the delay. In the present experiment, the goal was to measure the effect of the delay on the probability of revisiting predelay choices during postdelay testing. Errors made prior to the delay, as well as those involving revisits to arms that were initially visited following the delay, were excluded from the analysis. Because of the logical structure of the radial maze task, fewer choices follow the delay in the late $P O I$ conditions than in the early $P O I$ conditions. Thus, the early $P O I$ conditions include a greater number of chances to make errors following the delay. On the other hand, in the late $P O I$ conditions more potential sources of error exist than in the early $P O I$ conditions, because more arms are visited prior to the delay in the late delay conditions.

Because of these properties of the task, a new performance measure was developed. It measures the deviation from expected performance of the probability of revisiting predelay choices during postdelay testing. The expected value was generated by treating the control trials as if a delay had been interpolated at the same point as it was in the experimental condition. This resulted in an observed deviation from control (DFC) of the empirical probability of revisiting predelay choices. Specifically,

$$
D F C=(V e / O e)-(V c / O c),
$$

where $V e$ is the number of revisits to predelay choices during postdelay testing, and $O e$ is the number of arms chosen correctly prior to the delay (arms available to revisit after the delay or "opportunities" to make errors). $V c$ and $O c$ are the corresponding values for the control trials, when treated as if a delay had been interpolated. The value of $V c / O c$ depends on which $P O I$ condition is being evaluated; the control trials were treated as if a delay had been inserted at the same point as it was in the $P O I$ condition being evaluated. $D F C$ was determined for each $P O I$ condition in each block by subtracting $V c /$ $O c$ for each subject's two control trials of that block from $V e / O e$ for its two experimental (delay) trials.

The advantage of this measure is that it compares performance in each condition with performance in a control condition that is equivalent in every way except for the presence of the delay. The same number of choices are made prior to the choice of interest in the control condition as in the experimental (delay) condition, and the same number of correct choices remain to be made following that choice (with the exception of rare trials that included an error prior to the delay; these trials are the reason for separate divisors in Equation 1). Thus, differences in choices remaining to be made following the delay and the potential sources of errors in the different $P O I$ conditions are equated by comparing errors in each $P O I$ condition with errors in a control that is equivalent with regard to these factors. The $D F C$ measure allows comparisons of the information lost from memory 
as a result of the delay in the various conditions, as reflected by the increase in the percentage of predelay choices that are revisited.

Serial position curves for each POI condition were obtained by dividing the number of revisits to arms initially chosen at each predelay serial position by the number of trials in which the arm visited at that serial position was chosen correctly.

\section{Results}

The mean number of choices taken to finish the maze as a function of $P O I$ is shown in Figure 1. This measure is useful for comparing conditions that included a delay to the no-delay control condition as well as for comparing the present results with those of other investigators. In order to determine if the 15-min delay had an effect on performance, a repeated measures analysis of variance (ANOVA) was performed. In this and all other ANOVAS reported, the $p<.05$ level of significance was used. Data from the five delay conditions were combined and compared to the no-delay control condition. This analysis revealed that accuracy was reduced by the presence of a delay, $F(1,8)=19.6$, $p<.01$.

The mean $D F C$ in the various delay interpolation conditions is shown in Figure 2 . This measure allows comparison of the reduction in performance due to the presence of the delay in the various $P O I$ conditions. The mean of the control values $(V c / O c$ in Equation 1) used in generating these $D F C$ scores was $.22, .12, .09, .06$, and .05 for the $P O I=2,4,6,8$, and 10 conditions, respec-

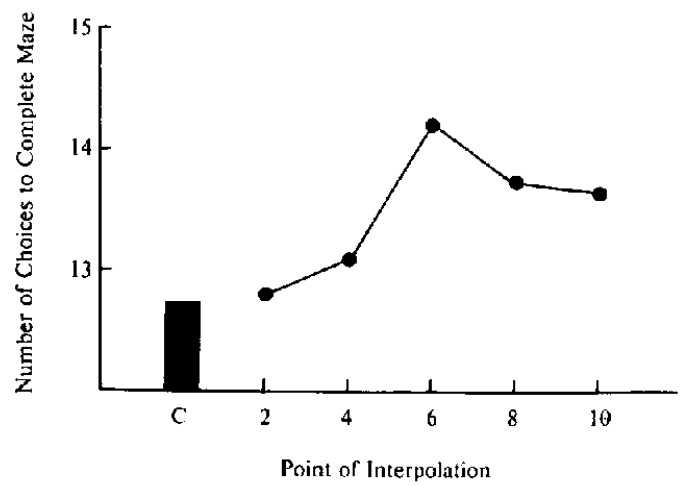

Figure 1. The number of choices taken to complete the maze in the control (C) and delay conditions of Experiment 1. (Point of interpolation refers to the choice number that preceded the delay.)

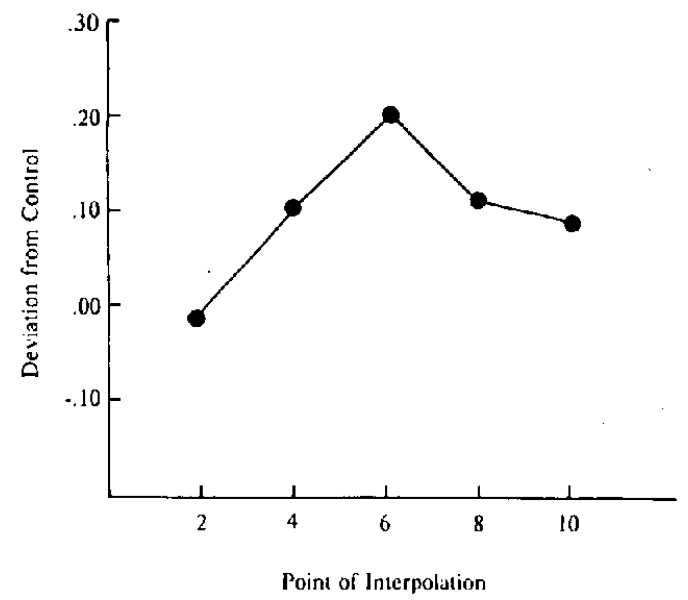

Figure 2. Deviation from control $(D F C)$ in the point-ofinterpolation $(P O I)$ conditions of Experiment 1. (DFC is an index of the disruptive effect that the delay had on performance, as measured by a comparison of accuracy. in the delay and control conditions. [See text for explanation.] $P O I$ refers to the choice number that preceded the delay.)

tively. Visual inspection of the data indicates that the delay had the greatest effect when it was interpolated in the middle of the choice sequence and less of an effect when it occurred either earlier or later. A repeated measures ANOVA for polynomial trends was conducted, using the $D F C$ scores of each rat in each block. It revealed a significant quadratic trend for the effect of POI, $F(1,32)=6.01, p<$ .02 ), with no other trends being significant.

The probability of revisiting predelay choices is shown according to choice number in Figure 3. These data were combined into bins of two serial positions to provide a more stable measure. Because of this, serial position data from the $P O I=2$ condition are not presented or discussed. The small number of errors in each cell did not allow for statistical evaluation of these curves. However, they suggest that the recency effect (i.e., the tendency for earlier choices to be revisited more often) is restricted to, or more pronounced in, the early and middle $P O I$ conditions.

\section{Discussion}

The finding of primary interest in the present experiment is that the effect of a 15 min delay on rats' ability to avoid arms chosen prior to the delay varies systematically 


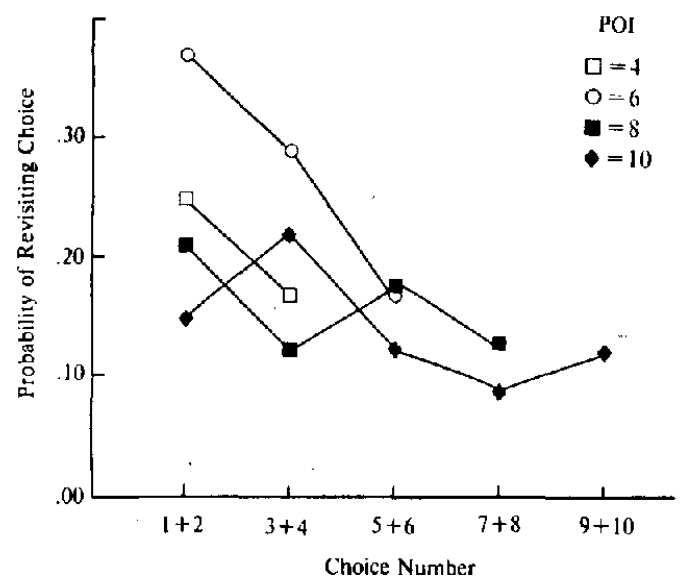

Figure 3. Serial position curves from Experiment 1 for the delay conditions. (Each point represents the probability of revisiting the arm corresponding to the predelay choice number shown. Choice numbers are collapsed into bins of two choices each. Symbols indicate the point-ofinterpolation $[P O I]$ condition. [Condition $P O I=2$ is not shown.])

with the point-of-delay interpolation. The delay had the greatest effect when it was interpolated in the middle of the choice sequence and less of an effect when interpolated either earlier or later. The inverted U-shaped function relating $P O I$ and the disruptive effect of the delay on performance $(D F C)$ is evidence for a two-process mechanism, with the positive slope during early delay conditions indicating the use of retrospective coding and the negative slope during late delay conditions indicating prospective coding. According to this dual-code hypothesis, the fact that a delay has less of an effect early in the choice sequence than it does in the $P O I=6$ condition is due to representations of a greater number of previously visited arms being maintained during the delay in the $P O I=6$ condition than in the earlier $P O I$ conditions. When the delay occurs later in the choice sequence, however, choices are guided by representations of the arms that remain to be visited rather than the previously visited arms. This results in the delay's having less of an effect.

The serial position curves provide additional support for this hypothesis. It appears that a systematic relation between the serial position of a predelay choice and the probability of revisiting that choice existed only in the $P O I=4$ and $P O I=6$ conditions. Such a relation could exist only if the previously visited arms, rather than the to-be-visited arms, are represented in memory.

Riley et al. (1981) suggested that the reduction of memory load is an important determinant of the situational specificity of prospective coding. The results of Experiment 1 suggest that a reduction in memory load may play a role even within the same task. The type of memory code used during a particular trial seems to depend on the amount of information contained in each of the code types, with the most efficient code being used. It appears that rats practice a kind of cognitive economy by changing their representations of the world in order to keep information load at a minimum.

\section{Experiment 2: Variation of Retention Interval Length}

The second experiment had two purposes. The first was to provide a replication of the effect of $P O I$ found in Experiment 1. To this end, half of the experimental conditions in Experiment 2 consisted of 15-min delays interpolated following the 2 nd, 6 th, or 10 th choice.

The second purpose was to explore the effects of delay length on the two different memory codes implied by the results of Experiment 1. An important step in the analysis of any memory process is an examination of its time course. To the extent that retrospective and prospective memories are processed and stored differently, it is not unreasonable to expect that they have different time courses. Such a result would be further evidence that two processes are operating. If differences were found, then manipulations of delay length would provide a method for controlling and isolating the two memory codes. In addition, any changes in the shape of the function relating $P O I$ and $D F C$ with delay length would help eliminate the possiblity that the shape of the function found in Experiment 1 was an artifact of the procedure or the $D F C$ measure.

\section{Method}

Subjects and apparatus. The 9 rats that completed Experiment 1 served as subjects. The apparatus was the same as in Experiment 1. 
Procedure. Prior to Experiment 2, two manipulations were conducted that are not reported. The first involved rotating the maze during a 15 -min delay as a check for the use of intramaze cues. Rotation had no effect on choice accuracy. The second manipulation was an inappropriate forced-choice procedure. Doors were placed at the entrance to a random set of 2,6 , or 10 arms prior to each trial, and the subject was allowed to deplete the food on the unblocked arms. After a 15-min delay, the subject returned to an unblocked maze to finish the trial. The data from this experiment could not be evaluated in terms of the effect of $P O I$ due to the absence of a nodelay control condition.

Experiment 2 was conducted in the same manner as Experiment 1, except that only three POI conditions were used, and the length of the delay was either $15 \mathrm{~min}$ or $60 \mathrm{~min}$. The seven trial types, six delay conditions, and a no-delay control condition were presented in a random order in each of three blocks. Control trials consisted of uninterrupted runs in the maze. The six experimental trials had a delay interpolated after the 2 nd, 6 th, or 10th choice. Delay intervals of either $15 \mathrm{~min}$ or $60 \mathrm{~min}$ were combined factorially with point of interpolation. Data were assessed in the same manner as in Experiment 1, with each delay trial being compared with the control trial from the same block to produce the $D F C$ measure.

\section{Results}

The mean number of choices taken to finish the maze as a function of $P O I$ and delay interval is shown in Figure 4. A repeated measures ANOVA (POI $\times$ Delay Length $\times$ Rats) was performed on the number of choices taken to finish the maze in each delay condition of each block. It revealed that more choices were required to finish the maze after

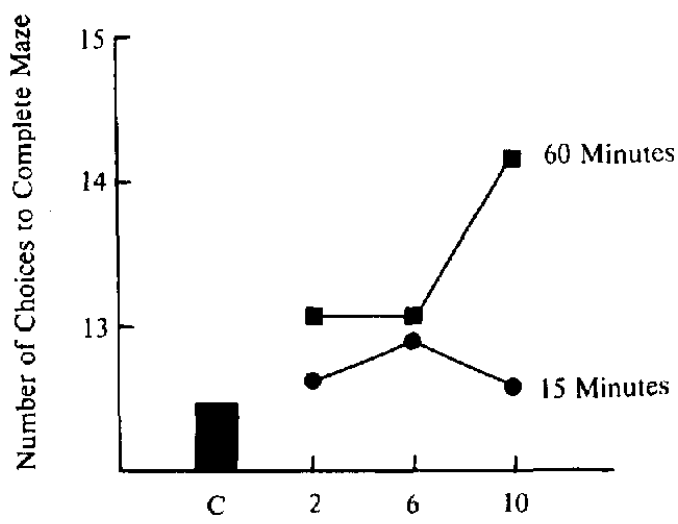

Point of Interpolation

Figure 4. The number of choices taken to finish the maze in the control (C) and delay conditions of Experiment 2. (Point of interpolation refers to the choice number that preceded the delay.)

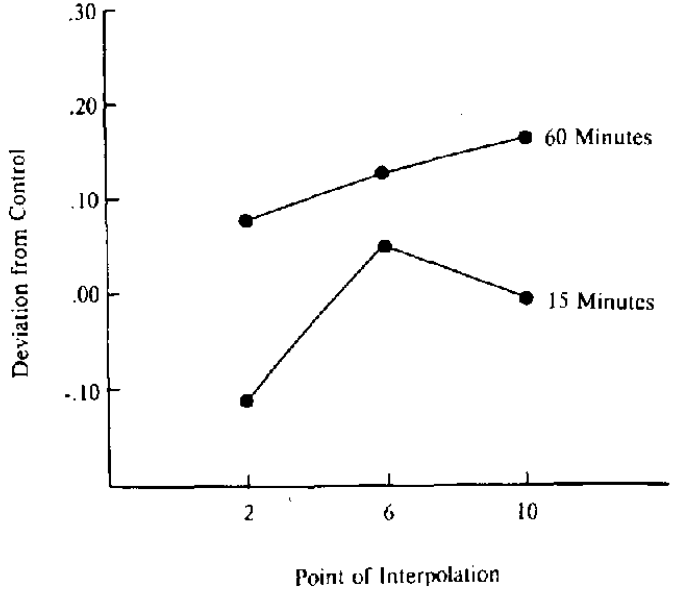

Figure 5. Deviation from control in the point-of-interpolation conditions of Experiment 2. (Point of interpolation refers to the choice number that preceded the delay.)

a delay of $60 \mathrm{~min}$ than after a 15-min delay, $F(1,8)=11.9, p<.01$.

The mean $D F C$ for each $P O I$ condition is shown in Figure 5. The means of the control values $(V c / O c$ in Equation 1) used in generating these $D F C$ scores were $.18, .07$, and .04 for the $P O I=2,6$, and 10 conditions, respectively. Choice accuracy was affected by delay length according to this measure as well, $F(1,8)=21.0, p<.002$. Analysis of the 15-min delay conditions revealed significant linear, $F(1,16)=4.7, p<.045$, and quadratic, $F(1,16)=6.1, p<.025$, trends. Analysis of the $60-\mathrm{min}$ delay conditions revealed no significant trends.

The serial position curves from the $P O I=$ 6 and $P O I=10$ conditions are shown in Figure 6 . Visual inspection indicates that a recency effect existed in the $P O I=6$ condition, indicating retrospective coding, regardless of delay length. However, it appears that a recency effect was obtained in the $P O I=$ 10 condition with a 60 -min delay, but not with a 15-min delay. The apparent existence of a recency effect in the $P O I=10$ condition following a $60-\mathrm{min}$ delay, but not following a 15-min delay, is supported by the results of a Wilcoxon signed-rank test, which was performed on the difference between the number of errors from the first five and last five predelay choices in the two $P O I=10$ conditions. A significantly greater proportion of the errors were from the early choices in the 

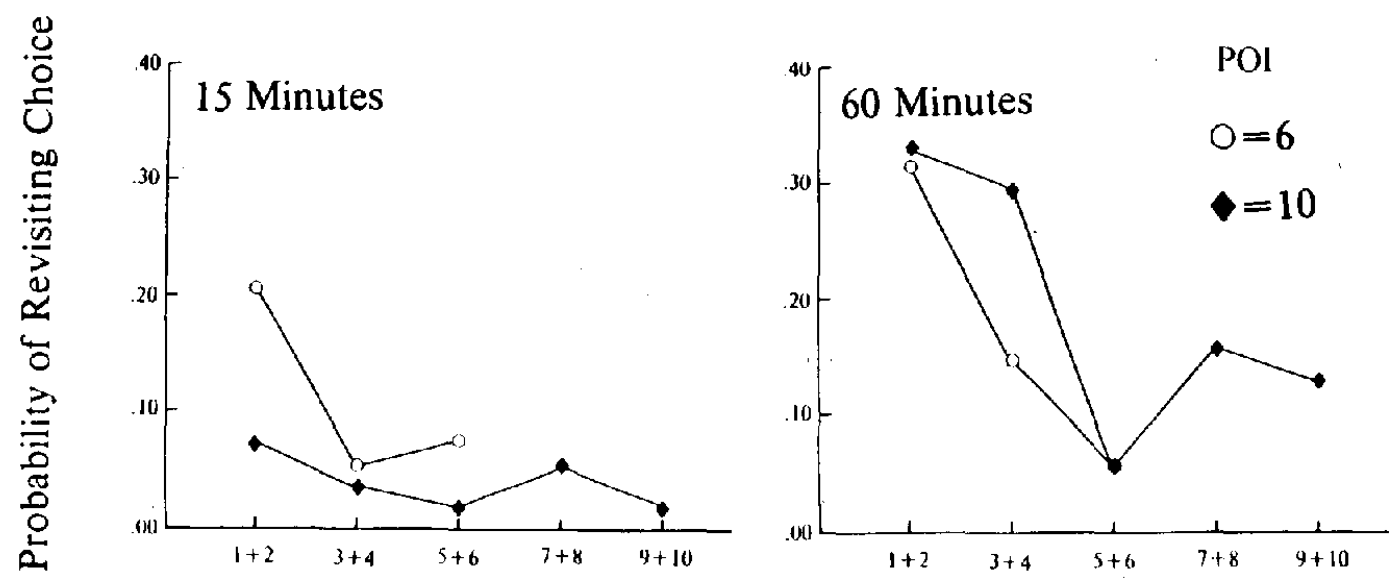

\section{Choice Number}

Figure 6. Serial position curves from the $P O I=6$ and $P O I=10$ delay conditions of Experiment 2. (Data from 15-min delay conditions are shown in the left panel. Data from the 60-min delay conditions are shown in the right panel. Each point represents the empirical probability of revisiting the arm corresponding to the predelay choice number shown. Choice numbers are collapsed into bins of two choices each. Symbols indicate the point-of-interpolation $[P O I]$ condition. [Conditions with $P O I=2$ are not shown.])

60-min delay condition than in the 15-min condition $(p<.05)$.

\section{Discussion}

This experiment replicates the finding of Experiment 1 that a $15-\mathrm{min}$ delay has the greatest effect when interpolated in the middle of the choice sequence in a 12-arm radial maze. The data support the hypothesis that either retrospective or prospective memory codes can be used to mediate a 15-min delay. It should be noted, however, that the function relating $P O I$ and $D F C$ in the 15 -min delay conditions includes a negative $D F C$ value in the $P O I=2$ condition (Figure 5). This may have resulted from the large amount of recent experience with 15-min delays that subjects had had by the time the present experiment was conducted. Despite this anomaly, the quadratic component of the function relating $P O I$ and $D F C$ provides further support for the conclusions of Experiment 1.

In the present experiment, a significant linear trend was also found among the 15min POI conditions. One possible explanation for this is an increased reliance on retrospective coding due to the 60 -min delay conditions (see below). However, this effect is largely due to the negative $D F C$ found in the $P O I=2$, 15-min delay condition, a fact that complicates its interpretation.

Inspection of Figure 5 suggests that, in the 60-min delay conditions, the delay had its greatest effect when it occurred following the 10th choice. Such a pattern of results indicates the use of retrospective memory. The lack of statistical support for a linear trend in these data does not allow them to be taken as strong support for retrospective coding, however. The 60-min delay produced large individual differences in the $P O I=10$ condition, with several rats making many errors while others made few. Thus, it may be that these conditions exaggerated individual differences in coding.

Support for the notion that retrospective coding was used by at least some subjects in the $P O I=10,60-\mathrm{min}$ delay condition comes from the serial position curves. Most errors that occurred in this condition were revisits to arms chosen early in the choice sequence. As has been argued, this result implies retrospective coding. It appears that in at least some of our subjects, retrospective information was used following a 60 -min delay even though prospective coding was used following a 15 min-delay. If rats use prospective codes 
following a $15-\mathrm{min}$ delay in the $P O I=10$ condition (as indicated by the $D F C$ data), but use retrospective codes following a 60 min delay (as indicated by the serial position data), then both kinds of information must be accessible early in the delay.

\section{Experiment 3: Free Versus Forced Predelay Choices}

In the two previous experiments, rats were allowed to choose freely from the 12 arms of the maze. This procedure allows the possibility of turning biases or response strategies (cf. Bond, Cook, \& Lamb, 1981; Suzuki et al., 1980; Yoerg \& Kamil, 1982). For example, rats often tend to turn in a consistent direction following each choice and then visit an adjacent or nearby arm. Brown and Cook (1982) reported detectable response biases among some of the same subjects that were used in Experiments 1 and 2. The effect of such response biases on performance in the radial maze is largely unknown. Brown and Cook found no correlation between the degree of response bias of a subject and its accuracy. It is possible, however, that the existence of such response biases contributes to the effect of POI. A response pattern interrupted in the middle may be harder to overcome than one interrupted either early or late. Therefore, a forced-choice procedure was used in Experiment 3. The arms chosen prior to the delay were determined randomly by the experimenter rather than by the subject. This procedure rules out any contribution of response biases. In addition, the use of a forcedchoice procedure provides greater control over the relation between the number of arms visited (or remaining) and the amount of information in memory. Conditions with free choices prior to the delay were also included in the experimental design to allow comparison of performance in the two procedures.

A second modification of the procedure was designed to increase the similarity of the control trials to the POI conditions. In the previous experiments, the data from each delay condition were compared to the data from the control trial. The control data were treated as if a delay had occurred. A problem with this method of evaluating the effect of the delay is that, in addition to the presence of the delay, the delay conditions include an interruption of behavior at a particular POI, whereas the control condition does not. Therefore, in the present experiment, performance in each delay condition was evaluated relative to performance in a control condition in which the doors to the arms were closed for $5 \mathrm{~s}$ at the same point in the choice sequence as the delay occurred in the relevant $P O I$ condition.

\section{Method}

Subjects. The subjects were 21 naive male LongEvans hooded rats, approximately 6 months of age. They were maintained in the same manner as the rats in the previous experiments. Trials occurred during the first 4 hours of the light phase.

Apparatus. The maze was the same as in the previous experiments, except that the single guillotine door was replaced by individual doors for each arm. These could be raised simultancously or individually by the experimenter. The maze was located in a different room than that used in Experiments 1 and 2. Extramaze cues included the rack of home cages, a table and chair used by the experimenter and located approximately $1 \mathrm{~m}$ from the maze, and a variety of posters placed on the walls of the room. Holding cages were located above the home cages.

Procedure. Initial training consisted of placing the rats, in groups of three, on the maze with pellets located along each arm and in the food cups. Starting with the third session, subjects were placed in the maze individually until they had depleted the food, made 20 choices, or until $20 \mathrm{~min}$ had elapsed. The number of pellets on the arms was reduced over trials until subjects completed the maze with two pellets in each food cup. Training continued until each subject had completed the maze within 20 choices for 15 trials. This took a mean of 24.7 trials (range: $17-40$ ).

The rats were then tested in three blocks of 20 trials. Trials varied according to three factors. An interruption occurred during the trial following either the 2nd, 4th, 6 th, 8th, or 10th correct choice. Half of the trials consisted of free choices, whereas the other half consisted of forced choices, prior to trial interruption. In the forced-choice conditions, the subject was placed in the maze, and the door to a single arm was opened approximately $3 \mathrm{~s}$ later. While the rat visited that arm, a second arm was opened. Each arm was closed during the subject's visit to the following arm. Between each choice, the arm that had just been chosen and the arm scheduled to be chosen next were available. The sequence of choices was determined by a random sampling without replacement procedure. Free-choice conditions were run in the same manner as in Experiments 1 and 2, except for the requirement that, as in the forced-choice conditions, the scheduled number of correct choices be made. If a predelay choice was incorrect, additional predelay choice(s) were allowed.

The third factor was the length of the "delay." Half of the trials contained 15-min delays. During the other half of the trials, the doors to the arms were closed for $5 \mathrm{~s}$. 
In 15-min delay conditions, the rat was removed from the maze and placed in a holding cage. During 5-s "delay" conditions, the rat remained on the center platform while the doors were closed. Following the delay, the doors were opened simultaneously, and the rat chose freely from the arms until all 12 arms had been visited or 20 total choices had been made, including predelay choices.

Each of the three blocks contained one trial each of 20 different types (Five POIs $\times$ Free vs. Forced Predelay Choices $\times 15$-min Delay vs. 5-s "Delay"), executed in a random order.' In an effort to increase error rates, there was a slight change in conditions during the second block. During this block, the illumination level in the experimental room was reduced by using two $25-\mathrm{W}$ light bulbs instead of the fluorescent light fixtures used during Blocks 1 and 3 of the experiment. One bulb was above the center of the maze, and the other was directed at the experimenter's work station. Because this manipulation had no effect (see below), it was abandoned following Block 2.

\section{Results}

The total number of revisits to choices made prior to the delay (in all trial types) was as follows: Block $1=95$; Block $2=63$; Block $3=61$. This indicates that performance was not affected by the reduction in illumination during Block 2. Because of this, the data from the three blocks were combined to provide more stable $D F C$ measures. For each rat, a $D F C$ score was calculated for each $P O I$, separately for the free- and forcedchoice conditions. Data from the 5-s delay conditions were used as the control factor $(V c / O c$ in Equation 1). In the free-choice conditions, the mean proportion of predelay choices revisited following the 5-s delay was $.040, .028, .021, .004$, and .002 for the $P O I$ $=2,4,6,8$, and 10 conditions, respectively. The corresponding scores from the forcedchoice conditions were $.040, .016, .008, .012$, and .006. The mean $D F C$ scores are shown in Figure 7.

Repeated measures ANOVAs for trend were conducted on the $D F C$ scores for free- and forced-choice trials. Although a quadratic trend is suggested by the data from freechoice conditions, neither a significant linear trend, $F(1,80)<1$, nor quadratic trend, $F(1$, $80)=1.47$, was found. The data from forcedchoice conditions contained a significant quadratic trend, $F(1,80)=4.15, p<.05$, but no linear trend, $F(1,80)<1$.

The probability of revisits to predelay choices as a function of choice number in

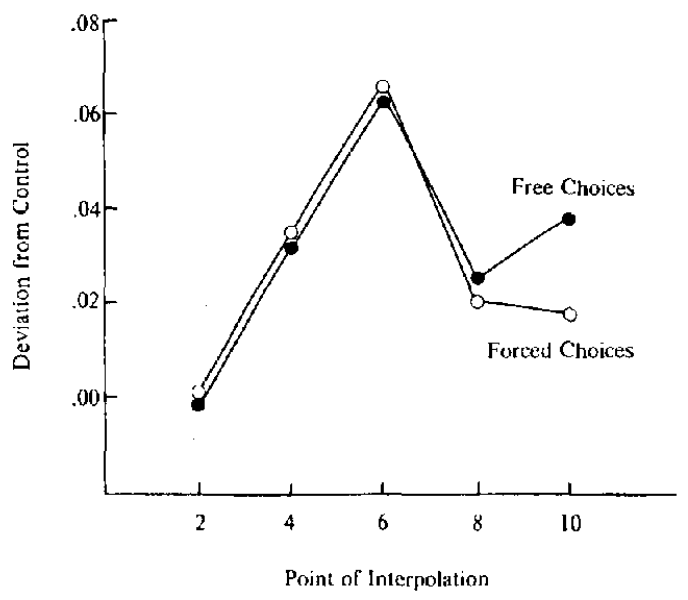

Figure 7. Deviation from control in the point-of-interpolation conditions of Experiment 3. (Point of interpolation refers to the choice number that preceded the delay.)

the 15-min delay conditions is shown in Figure 8. In trials with free choices, only the $P O I=6$ condition shows the recency effect indicated by Experiments 1 and 2 . In the forced-choice conditions, a recency effect is apparent in the $P O I=4$ and $P O I=6$ conditions.

\section{Discussion}

The present experiment replicates the relation between $P O I$ and $D F C$ found in the earlier experiments using a forced-choice procedure. This eliminates explanations of the finding in terms of differential use of response patterns prior to the delay. In the forced-choice procedure, subjects cannot visit arms in a systematic order that may facilitate the consistent encoding of multiple arms as single units, or "chunks." This increases the plausibility of the assumption that the amount of information retained in memory is a function of the number of arms visited prior to the delay or the number remaining to be visited.

Although the data from the free-choice conditions were too variable to provide sta-

\footnotetext{
${ }^{1}$ In an effort to prepare the rats for Experiment 4, an additional trial was conducted following the completion of each block of Experiment 3. This trial contained one of the conditions to be used in Experiment 4, chosen randomly, and was conducted in the same manner as the trials of Experiment 4.
} 

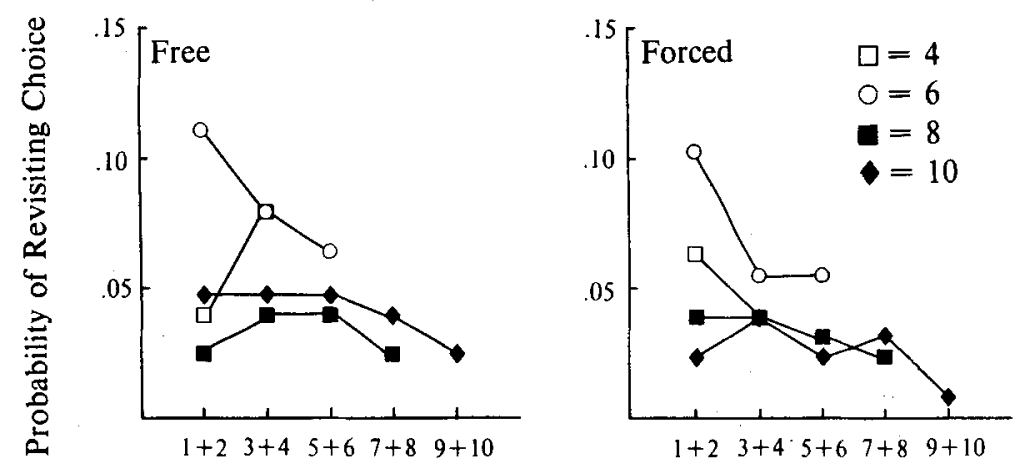

Choice Number

Figure 8. Serial position curves from the 15-min delay conditions of Experiment 3. (Data from the freechoice conditions are shown in the left panel. Data from the forced-choice conditions are shown in the right panel. Each point represents the empirical probability of revisiting the arm corresponding to the predelay choice number shown. Choice numbers are collapsed into bins of two choices each. Symbols indicate the point-of-interpolation $[P O I]$ condition. [Conditions with $P O I=2$ are not shown.])

tistical support, the pattern of results is consistent with the results of Experiments 1 and 2. Inspection of the data from the three blocks suggested that the inverted U-shaped $P O I$ function was present during the first block, but $D F C$ decreased in the $P O I=6$ condition during Blocks 2 and 3, resulting in a floor effect.

The serial position curves found in this experiment are consistent with the idea that retrospective coding was used in the $P O I=$ 4 and $P O I=6$ conditions, but not in the $\mathrm{POI}=8$ or $P O I=10$ conditions. The one curve that is not consistent with this notion is from the $P O I=4$, free-choice condition. In that case, the later predelay choices were revisited more often than the earlier predelay choices. The shape of the other seven serial position curves appears to agree with the prediction that early and middle $P O I$ conditions produce a negatively sloped serial position curve, whereas the late $P O I$ conditions show no relation between serial position and probability of revisits.

\section{Experiment 4: Two-Alternative Forced- Choice Tests}

The final experiment is directed at two issues pertaining to the dual-code explanation of the results of Experiments 1,2, and 3 . The first concerns the properties of the $D F C$ measure. Use of the $D F C$ measure requires several assumptions, the most important of which is that the effect of the delay should be evaluated in terms of the increase in the proportion of predelay choices that are revisited as a result of the delay rather than in terms of the increase in the absolute number of errors. The $D F C$ score is superior to measures that compare absolute numbers of errors because the probability of making errors by chance differs as a function of $P O I$. It would be improper to compare differences in numbers of errors in different $P O I$ conditions because the addition of, for example, a single extra error with a delay represents a greater loss of information when only 2 possible errors exist $(P O I=2$ condition) than when 6 or 10 possible errors exist. It is unclear, however, whether a $D F C$ of $10 \%$, for example, reflects an equivalent information loss in early $P O I$ conditions (in which a difference of a single error produces a large $D F C$ ) and in late $P O I$ conditions (in which a difference of a single error has less of an effect). In essence, it is uncertain whether the $D F C$ metric is linear with respect to the information loss that it is designed to measure.

The second issue concerns an alternative to the dual-code hypothesis. This hypothesis agrees with the dual-code hypothesis that the increase in $D F C$ seen in the early POI conditions is the result of the increasing number 
of predelay choices that must be remembered as the $P O I$ comes later (i.e., retrospective coding). The decrease in $D F C$ seen in the late $P O I$ conditions, however, is not attributed to prospective coding. Rather, the rat is proposed to be sensitive to the changes in the probability of making errors by chance as a function of how many arms have been visited. As a result of this sensitivity, there are differences in the criterion that the rat uses in making choices, depending on the $P O I$. Following an early $P O I$, the animal can be relatively confident that any particular arm is correct because few arms have been visited. Following a late $P O I$, however, the probability of making errors is high because more arms have been visited. This might result in the animal's adjusting a choice criterion, visiting arms following a late $P O I$ only when a high level of confidence exists. Such a "criterion shift" would result in higher accuracy following a late POI. Thus, the combination of retrospective coding and differences in a choice criterion could have produced the pattern of results seen in the previous experiments.

The present experiment used two-alternative forced-choice (2AFC) tests to measure the effect of the delay in the different $P O I$ conditions. Following the delay, rats were given a choice between a single correct (novel) arm and a single incorrect (previously visited) arm. This procedure addresses both of the issues discussed above. By using the 2AFC test, performance can be measured in terms of percent correct, and results from different $P O I$ conditions compared directly. No comparison of delay and control conditions (the $D F C$ measure) is necessary.

This procedure also allows the dual-code and criterion-shift hypotheses to be contrasted. In the 2AFC tests, the probability of a random choice being correct is the same in every condition ( $50 \%)$. Because the proportion of available arms is the same regardless of $P O I$, no shift in criterion should occur. Hence a replication of the pattern of results found in the previous experiments with this testing procedure would validate the conclusions drawn based on the $D F C$ scores in Experiments 1, 2, and 3 and provide evidence against the criterion-shift hypothesis.

\section{Method}

Subjects and apparatus. The same subjects and apparatus were used as in Experiment 3.

Procedure. The experiment consisted of 12 blocks. Each block contained five conditions, presented in a random order. The condition was specified by the pointof-delay interpolation, with the delay occurring after either the 2nd, 4th, 6th, 8th, or 10th choice.

Prior to the delay, trials were run in the same manner as the forced-choice trials of Experiment 3. Following the last predelay choice, the rat was placed in a holding cage for $15 \mathrm{~min}$. After the delay, it was placed in the center of the maze, and the doors to two arms were opened simultaneously. One of these arms had been visited prior to the delay and contained no food. The other arm had not been visited prior to the delay and contained two food pellets. The subject was allowed to visit one of the two arms, and the doors were closed upon its return to the center. A second 2AFC test occurred immediately. Following the second test, the rat was returned to its home cage. The set of arms visited prior to the delay, as well as the correct arms presented in the tests, were determined by a random sampling without replacement procedure. The incorrect arms presented in the tests were chosen randomly from among the arms chosen before the delay, with the constraint that the same arm did not serve as the incorrect choice in both tests of a trial.

\section{Results}

Preliminary analyses revealed that very few errors were made during the second half of the experiment. The experiment was therefore divided into two halves (six blocks per half). A repeated measures ANOVA (Rats $X$ $P O I \times$ Half of the Experiment) using the number of errors in each trial revealed a significant interaction between halves of the experiment and $P O I, F(4,80)=3.3, p<$ .025 , due to the lack of errors during the second half of the experiment. Therefore, subsequent analyses were carried out separately for the first six and last six blocks of the experiment.

The mean percentages of possible errors made in the various $P O I$ conditions during the first and second halves of the experiment are shown in Figure 9. Repeated measures ANOVAs for trend were conducted on both sets of data. Results from the first six blocks included a significant quadradric component, $F(1,80)=10.8, p<.002$, but no linear component $(F<1)$. Thus, subjects made more errors when the delay was in the middle of the choice sequence and fewer errors when 


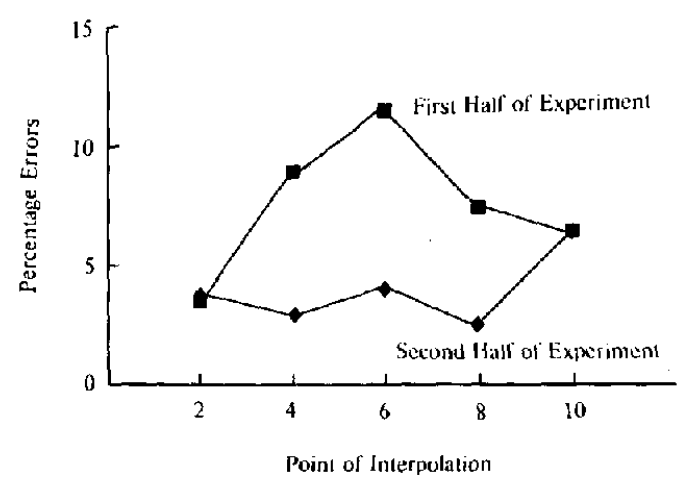

Figure 9. Mean percentage of errors made in the twoalternative forced-choice tests for the delay conditions of Experiment 4. (Point of interpolation refers to the choice number that preceded the delay.)

the delay occurred either early or late in the choice sequence. Data from the last six blocks contained no significant trends.

The serial position curves for the $P O I$ conditions during the first half of the experiment are shown in Figure 10. Each point represents the number of revisits to that choice number divided by the number of tests that included that choice number. The $N s$ were approximately equal across serial positions within each $P O I$ condition. A greater tendency to make errors to earlier chosen arms is suggested only in the $P O I=6$ condition. Serial position curves from the second half of the experiment are not shown due to the paucity of errors.

\section{Discussion}

The results from the present experiment provide additional support for the conclusions drawn as a result of the first three experiments. During the first six blocks, rats made more errors in a two-alternative forced-choice test when they had visited six arms prior to the delay than they did when they had visited either fewer arms or more arms. The fact that the pattern of results obtained with the 2AFC tests agrees with that obtained in the previous experiments further validates the $D F C$ measure. An improvement in performance resulted in a floor effect during the second half of the experiment.

The present pattern of results cannot be readily explained by the criterion-shift hy- pothesis, because information about the number or proportion of arms visited is irrelevant in the 2AFC tests. Even if such a shift in choice criterion occurs in the standard radial maze task, due to the increasing probability of errors over the course of each trial, it is unlikely that this could have affected performance in the present experiment, because the probability of making an error by chance was $50 \%$ in all conditions. $2 \mathrm{AFC}$ tests have been used in detection tasks to measure performance independently of any effects of bias or criterion (cf. Swets, Tanner, \& Birdsall, 1961). The present results may therefore be taken as evidence against the criterion-shift hypothesis.

It is of interest to compare the present results with those of Roberts and Smythe (1979; Experiment 3), who used a similar 2AFC testing procedure in an eight-arm maze. They found that rats were more likely to revisit arms chosen earlier in the choice sequence (i.e., there was a recency effect) when the test occurred following either the third, fifth, or seventh choice. Thus, in apparent contrast to the present experiments, evidence for retrospective coding was obtained under conditions that should seemingly en-

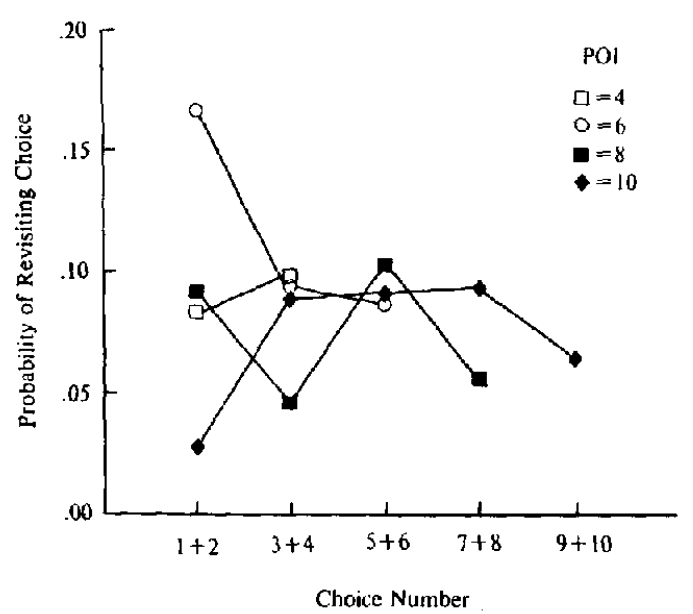

Figure 10. Serial position curves from the first half of Experiment 4. (Each point represents the empirical probability of revisiting the arm corresponding to the predelay choice numbers shown. Choice numbers are collapsed into bins of two choices each. Symbols indicate the point-of-interpolation [POI] condition. [Condition $P O I=$ 2 is not shown.]) 
courage prospective coding. There are several differences between the procedure of Roberts and Smythe and the current procedure that might account for this difference. First, the number of arms in the mazes differed. A large number of arms may be necessary to encourage the use of prospective codes. Second, Roberts and Smythe tested the rats immediately following their last scheduled choice, whereas the present procedure included a 15-min delay between predelay choices and the test. It is suggested below that a delay may be critical for allowing the use of prospective codes. Perhaps most intriguing is the difference in the amount of training that the animals received. Roberts and Smythe's animals received only seven trials in the maze prior to testing. The rats in Experiment 4 received approximately 85 trials in the maze prior to the experiment, and no animals in any of the present experiments were tested without substantial training. Certainly some amount of experience in the maze is necessary for the use of prospective memory. In order for anticipated arms to be coded, information about them must exist in some type of permanent, reference memory. Thus, it should be expected that relatively naive subjects would rely on retrospective coding.

\section{General Discussion}

The major finding in all four of the present experiments is the quadratic function that relates the point of interpolation of a $15-\mathrm{min}$ delay and choice accuracy. The delay disrupts the performance of rats in a 12-arm radial maze to a greater extent when it is interpolated in the middle of the choice sequence than when it is interpolated either early or late in the choice sequence. This is the case whether the predelay choices are chosen by the subject (Experiments $1 \& 2$ ) or are presented in a predetermined order (Experiments $3 \& 4)$. The effect is obtained if measured by comparing performance in a free-choice test following a 15-min delay with performance without a delay (Experiments 1, 2, and 3) or by two-alternative forced-choice tests (Experiment 4). Thus, the results are repeatable and obtained under a variety of conditions.

Associated with the effect of $P O I$ is a corresponding difference in the shape of the serial position curves. In order to summarize and compare the serial position effects obtained, the slopes of best-fit linear regression lines were calculated using the data shown in Figures 3,6,8, and 10. Among the conditions that included a 15-min delay, seven of nine of the $P O I=4$ and $P O I=6$ conditions had a slope of less than -.040 . On the other hand, all nine of the $P O I=8$ and $P O I=10$ conditions had slopes between -.020 and +.010 . Thus, when the delay is interpolated early or in the middle of the choice sequence, errors tend to be in the form of revisits to arms chosen earlier; that is, there is a recency effect. In the late $P O I$ conditions, however, there was little or no relation between the order in which predelay choices were made and the tendency of the subjects to revisit them.

These results support the hypothesis that rats use a flexible, dual-code memory system. Information can be represented in a form corresponding to previously presented information (retrospective coding) or in the form of information about anticipated events or actions (prospective coding). This flexibility seems to allow increased efficiency of cognitive processing in the sense that less information must be retained during a delay and/ or used following the delay as a result of it.

This notion of cognitive efficiency (cf. Riley et al, 1981) may have to be tempered by the results from the 1-hr delay conditions of Experiment 2. While further experiments will be required to clarify those data, they suggest that the representations in memory may be more complex than a simple retrospectiveto-prospective switching model would suggest. Although prospective memory is used following a 15 -min delay in the $P O I=10$ condition, rats seem to rely on retrospective memory following a 60 -min delay. It could be that representations can be translated back and forth between retrospective and prospective forms. Alternatively, perhaps both forms of memory are maintained, but they are differentially used. In either case, complicated and seemingly less efficient processes are involved than in a simple code switching model. The notion that dual coding functions to increase efficiency must also take into account whatever cognitive "cost" is incurred due to the 
process necessary for translating retrospective information into prospective information.

Although the present experiments were designed to determine the nature of the memory codes used in the radial maze, an alternative explanation for the pattern of accuracy results (the criterion-shift hypothesis) was proposed and tested in Experiment 4. This hypothesis attributes the negative slope of the $D F C$ measure in the late POI conditions to a change in the rats' choice criterion rather than to the use of prospective memory. As more arms are visited (and less food remains), the rat becomes more "careful" following a delay, and hence makes fewer errors as a result of the delay. The results of Experiment 4 argue against this hypothesis because the procedure used in that experiment is such that there is an equal probability of making errors by chance in all $P O I$ conditions. The logical structure of the $2 \mathrm{AFC}$ test does not encourage differences in choice criteria in different $P O I$ conditions. It should be noted, however, that because the rats in Experiment 4 had previously experienced conditions that the criterion-shift hypothesis would expect to encourage differences in choice criteria, they might have entered Experiment 4 with a tendency to use greater care in making choices in late $P O I$ conditions than in earlier $P O I$ conditions.

In addition to the results of Experiment 4, there are a number of reasons for favoring the dual-code hypothesis over the criterionshift hypothesis. First, the latter is a post hoc analysis. It relies on a process for which there is no evidence; rats must be sensitive to the number or proportion of choices that remain, and this must affect a choice criterion. On the other hand, it is clear that rats must retain information that allows them to successfully complete the maze and that this information is retained in some particular form (i.e., it is coded). There is abundant evidence that animals have the ability to retain information in a prospective form under some conditions (cf. Honig \& Thompson, 1982; Riley et al., 1981). Thus, the dualcode hypothesis is a more parsimonious explanation for the pattern of results than the criterion-shift hypothesis. Finally, the coding hypothesis, but not the criterion-shift hypoth- esis, predicts the serial position curves obtained in the present experiments.

Roitblat (1982) and Palmer (1978) have proposed metatheories of representation that help in clarifying the dual-code hypothesis. These authors argue that the same information can be represented in a variety of forms and that one must differentiate between the information contained in the representation and the form in which that information is represented. It could be argued that the prospective and retrospective codes used in the present task are "informationally equivalent" (Palmer, 1978). This is because it is always logically possible to translate knowledge of which arms have been previously visited into knowledge of which arms remain to be visited. It is not known, however, if rats make this translation or if prospective memory codes are formed independently of retrospective codes (see below). In any event, the form in which information is represented is quite distinct in the two cases. Assuming that the effect of a 15-min delay varies with the amount of information contained in the code, there is a greater amount of information represented in the code that is used when a delay occurs in the middle of the choice sequence than that used when few or many arms have been chosen.

When is the prospective code formed? There are a number of possibilities. First, it could be that the arms are encoded retrospectively as they are visited and that this retrospective code is transformed into a prospective code after the sixth choice is made or during the delay. An alternative possibility is that the rat maintains both a retrospective "list" of previously visited arms and a prospective list of to be visited arms while running the maze. Items (arms) are added to the former list and "checked off" the latter list as choices are made. The present experiments did not directly address the dynamics of code formation, but there is suggestive evidence with which to evaluate some possibilities. If the delay is necessary for a translation to prospective coding to occur, then there should be a recency effect among errors made late in control trials, indicating the use of retrospective coding. Data from the control trials of the first three experiments suggest the existence of such a recency effect, although 
the small number of errors in the control trials makes this line of evidence tenuous. A number of other experimenters have also reported serial position curves in the radial arm maze. Olton and Samuelson (1976) and Kessner and Novak (1982) reported robust recency effects in eight-arm mazes. Olton et al. (1977) found a similar effect in a 17-arm maze. As has been previously discussed, Roberts and Smythe (1979) found recency effects in an eight-arm maze using a procedure similar to that used in Experiment 4, but without a delay prior to the tests. These data suggest the possibility that a delay is required for the formation of prospective codes.

A second type of evidence that pertains to the issue of code formation comes from Experiment 2. In that experiment, subjects performed above chance following either a 15-min delay or a 60-min delay. If they were returned to the maze after $15 \mathrm{~min}$, they produced evidence of prospective coding. Following a 1-hr delay, however, no evidence for prospective coding was found, and the serial position curves suggested the use of retrospective coding. If retrospective codes were being used following a l-hr delay, the implication is that both kinds of codes were potentially available during the early portion of the delay. Two possible explanations for this exist. First, both codes may be simultaneously present in memory during the first $15 \mathrm{~min}$ of the delay. Alternatively, the rats may translate prospectively coded information into retrospective information at some point between $15 \mathrm{~min}$ and $1 \mathrm{hr}$ into the delay. In summary, the available evidence on the dynamics of coding suggests that (a) prospective coding requires the presence of a delay, and (b) under some conditions, retrospective codes may be retrieved and used even after the formation of prospective codes.

A number of previous experiments have provided evidence for the notion that codes in animal working memory are in the form of anticipated events. The present experiments extend this research by showing that evidence for both types of codes can be obtained in the same task. The ability to isolate and measure retrospective and prospective codes should allow their properties and determinants to be studied. The present pattern of results indicates that the code used in any particular situation depends, at least in part, on the memorial demands placed on the animal.

\section{References}

Atkinson, R. C., \& Shiffrin, R. M. (1968). Human memory: A proposed system and its control processes. The Psychology of Learning and Motivation, 2, 89195.

Bond, A. B., Cook, R. G., \& Lamb, M. R. (1981). Spatial memory and the performance of rats and pigeons in the radial-arm maze. Animal Learning \& Behavior, 9, 575-580.

Brown, M. F., \& Cook, R. G. (1982, April). Temporal and behavioral indices of radial maze performance in rats. Paper presented at the meeting of the Western Psychological Association, Sacramento, CA.

Glenberg, A. M., Bradiey, M. M., Thomas, A. K., \& Renzaglia, G. J. (1983). Studies of the long-term recency effect: Support for a contextually guided retrieval hypothesis. Joumal of Experimental Psychology: Learning, Memory, and Cognition, 9, 231-255.

Grant, D. S. (1981). Short-term memory in the pigeon. In N. E. Spear \& R. R. Miller (Eds.), Information processing in animals: Memory mechanisms (pp. 227256). Hillsdale, NJ: Erlbaum.

Honig, W. K. (1978). Studies of working memory in the pigeon. In S. H. Hulse, H. Fowler, \& W. W. Honig (Eds.), Cognitive processes in animal behavior (pp. 2II-248). Hillsdale, NJ: Erlbaum.

Honig, W. K. (1981). Working memory and the temporal map. In N. E. Spear \& R. R. Miller (Eds.), Information processing in animals: Memory mechanisms (pp. 167197). Hillsdale, NJ: Erlbaum.

Honig, W. K., \& Thompson, R. K. R. (1982). Retrospective and prospective processing in animal working memory. The Psychology of Learning and Motivation, $16,239-283$.

Honig, W. K., \& Wasserman, E. A. (1981). Performance of pigeons on delayed simple and conditional discriminations under equivalent training procedures. Learning and Motivation, 12, 149-170.

Kessner, R. P., \& Novak, J. M. (1982). Serial position curve in rats: Role of the dorsal hippocampus. Science, 218. 173-175.

Murdock, B. B., Jr. (1962). The serial position effect in free recall. Journal of Experimental Psychology, 64, 482-488.

Olton, D. S., \& Collison, C. (1979). Intramaze cues and "odor trails" fail to direct choice behavior on an elevated maze. Animal Learning \& Behavior, 7, 221223.

Olton, D. S., Collison, C., \& Werz, M. A. (1977). Spatial memory and radial arm maze performance of rats. Learning and Motivation, 8, 289-314.

Olton, D. S., \& Samuelson, R. J. (1976). Remembrance of places passed: Spatial memory in rats. Journal of Experimental Psychology: Animal Behavior Processes, 2, 97-116.

Palmer, S. E. (1978). Fundamental aspects of cognitive representation. In E. Rosch \& B. B. Lloyd (Eds.), Cognition and categorization (pp. 259-303). Hillsdale, NJ: Erlbaum.

Riley, D. A., Cook, R. G., \& Lamb, M. R. (1981). A 
classification and analysis of short term retention codes in pigeons. The Psychology of Learning and Motivation. $15,51-79$.

Roberts, W. A., \& Grant, D. S. (1976). Studies of shortterm memory in the pigeon using the delayed matchingto-sample procedure. In D. L. Medin, W. A. Roberts, \& R. T. Davis (Eds.), Processes of animal memory (pp. 79-112). Hillsdale, NJ: Erlbaum.

Roberts, W. A., \& Smythe, W. E. (1979). Memory for lists of spatial events in the rat. Learning and Motivation, 10. 313-336.

Roitblat, H. L. (1980). Codes and coding processes in pigeon short-term memory. Animal Learning \& Behavior, $8,34 \mid-351$.

Roitblat, H. L. (1982). The meaning of representation in animal memory. The Brain and Behavioral Sciences, $5,353-406$.

Sands, S. F., \& Wright, A. A. (1980). Serial probe recognition performance by a rhesus monkey and a human with 10 - and 20-item lists. Journal of Experimental Psychology: Animal Behavior Processes, 6 , 386-396.
Suzuki, S., Augerinos, G., \& Black, A. H. (1980). Stimulus control of spatial behavior on the eight-arm maze in rats. Learning and Motivation, 11, 1-18.

Swets, J. A., Tanner, W. P., \& Birdsall, T. G. (1961). Decision processes in perception. Psychological Review, $68,301-340$.

Wagner, A. R., \& Pfautz, P. L. (1978). A bowed serialposition function in habituation of sequential stimuli. Animal Learning \& Behavior, 6, 395-400.

Weisman, R. G., \& DiFranco, M. P. (1981). Testing models of delayed sequence discrimination in pigeons: Delay intervals and stimulus durations. Journal of Experimental Psychology: Animal Behavior Processes, 7. 413-424.

Yoerg, S. I., \& Kamil, A. C. (1982). Response strategies in the radial arm maze: Running around in circles. Animal Learning \& Behavior, 10, 530-534.

Received August 7, 1984 Revision received December 4, 1984

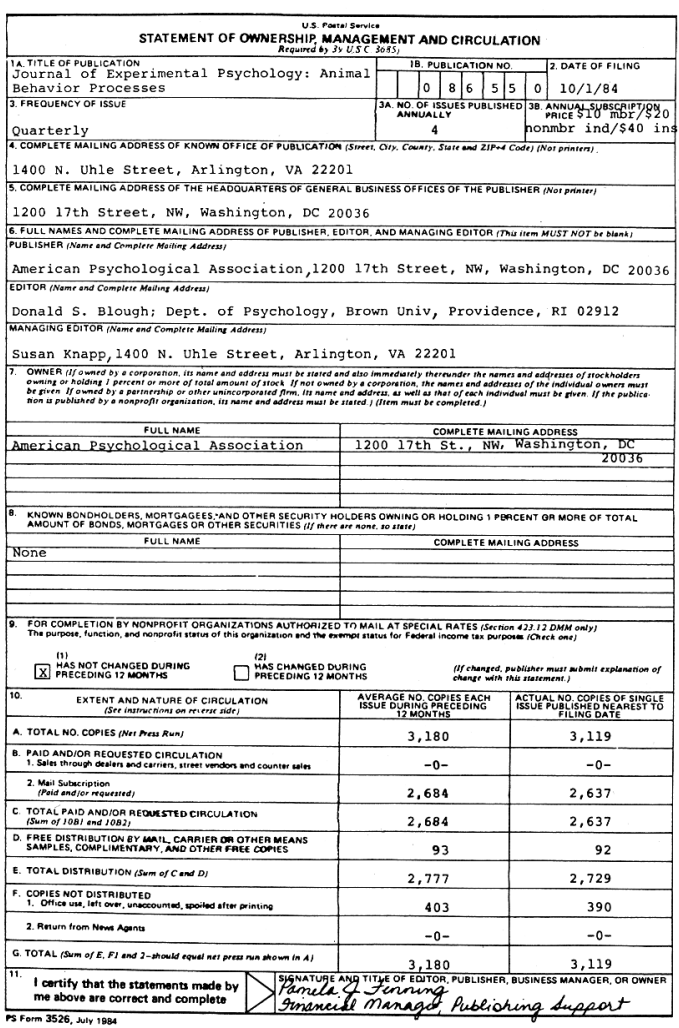

